\title{
ENTIRE VECTORS AND HOLOMORPHIC EXTENSION OF REPRESENTATIONS. II
}

\author{
BY
}

\section{RICHARD PENNEY}

\begin{abstract}
Let $G$ be a connected, simply connected Lie group and let $\boldsymbol{G}_{\boldsymbol{c}}$ be its complexification. Let $U$ be a unitary representation of $G$. The space of vectors $v$ at which $U$ is holomorphically extendible to $G_{c}$ is denoted $\mathcal{J}_{\infty}^{\omega}(U)$. In [9] we characterized those $U$ for which $\mathcal{H}_{\infty}^{\omega}$ is dense. In the present work we study $\mathcal{H}_{\infty}^{\omega}$ as a topological vector space, proving e.g., that $\mathcal{H}_{\infty}^{\omega}$ is a Montel space if $U$ is irreducible and $G$ is nilpotent. We prove a representation theorem for $\left(\mathcal{H}_{\infty}^{\omega}\right)^{\prime}$ which yields a Bergman kernel type theorem for $G$. As an application we give a necessary and sufficient condition for the set of holomorphic functions on certain solvmanifolds to separate points.
\end{abstract}

Introduction. In a previous paper [9] we showed that if $U$ is a representation of a type $R$ solvable Lie group $G$ in a Banach space $B$, then there is a dense set of vectors $v$ for which $g \rightarrow U_{g} v$ is extendible to a holomorphic map of the complexification $G_{c}$ of $G$ into $\stackrel{g}{B}$. Such vectors are called entire vectors and the space of such vectors is denoted by $\mathcal{H}_{\infty}^{\omega}(U)$. $\mathcal{H}_{\infty}^{\omega}$ is an invariant subspace and the restriction $U^{\omega}$ of $U$ to $\mathcal{H}_{\infty}^{\omega}$ possesses a holomorphic extension to $G_{c^{*}}$

One may put on $\mathcal{H}_{\infty}^{\omega}$ a Fréchet space structure defined via the family of norms

where $\Omega \subset G_{c}$ and is compact.

$$
\rho_{\mathbf{Q}}(v)=\sup _{\boldsymbol{z} \in \mathbf{Q}}\left\|U_{\boldsymbol{z}}^{\omega} v\right\|
$$

We refer to the above topology as the $\mathcal{H}_{\infty}^{\omega}$ topology. Under this topology $U^{\omega}$ is a continuous (in fact, holomorphic) representation of $G_{c}$ by continuous operators. (For the proofs of these facts see $\$$ I.)

In this paper we propose to study $U^{\omega}$ via the topological structure of $\mathcal{H}_{\infty}^{\omega}$. Our main interest is in the Hilbert space-unitary case.

In this case we prove that $\mathcal{H}_{\infty}^{\omega}$ is reflexive and, if $B$ is separable, so is $\mathcal{H}_{\infty}^{\omega}$. We also show that under a certain closely related, but weaker, topology $\mathcal{H}_{\infty}^{\omega}$ is a semi-Montel space. It turns out that if $G$ is nilpotent and $U$ is irreducible, the weaker topology in fact equals the $\mathcal{H}_{\infty}^{\omega}$ topology and $\mathcal{H}_{\infty}^{\omega}$ is a Montel space.

Our main tool is a representation theorem for the dual space $\left(\mathcal{H}_{\infty}^{\omega}\right)^{\prime}$ which states (in the unitary case) that every continuous linear functional is a finite

Received by the editors February 13, 1973 and, in revised form, July 23, 1973. AMS (MOS) subject classifications (1970). Primary 43A65, 22E45.

Key words and phrases. Analytic vector, Lie group, solvmanifold. 
linear combination of functionals of the form $v \rightarrow\left(U_{z}^{\omega} v, w\right)\left(z \in G_{c}, w \in B\right.$ fixed).

In a large number of cases (e.g. when $U$ is an induced representation) it can be shown that $\mathcal{H}_{\infty}^{\omega}$ can be identified with a space of entire functions on $G_{c}$ for which point evaluation is continuous. Our representation theorem yields a Bergman kernel type theorem for certain unbounded domains.

As an application of our results, we develop a necessary and sufficient condition for the entire functions on the complexification of a compact solvmanifold to separate points of the solvmanifold.

I. Throughout the sequel $G$ will denote a connected, simply connected, solvable, type $R$ Lie group and $\mathscr{L}$ will be its Lie algebra. Let $U$ be a fixed unitary representation of $G$ in a Hilbert space $\mathcal{H}$. Let $C^{\infty}(U)$ be the space of $C^{\infty}$ vectors for $U$ and, for $X \in \mathcal{L}$ and $v \in C^{\infty}(U)$, ler

$$
\partial U(X) v=\lim _{t \rightarrow 0}\left(\left(U \exp t X^{v-v) / t}\right)\right.
$$

The mapping $X \rightarrow \partial U(X)$ defines a representation of $\mathscr{W}$ by skew-symmetric operators which we extend to a representation of the universal enveloping algebra $\mathcal{U}$ of $\mathcal{L}$.

Let $X=\left(X_{1}, \cdots, X_{d}\right)$ be a fixed, ordered, Jordan-Hölder basis for $G$. Let $N$ denote the positive integers. If $n \in N^{d}$, let $X^{n}=X_{1}^{n_{1}} \ldots X_{d}^{n_{d}}$. Also if $n \in N$, let

$$
\{X\}^{n}=\left\{Y_{i} \cdots Y_{n} \mid Y_{i} \in\left\{X_{1} \cdots X_{d}\right\}, 1 \leq i \leq n\right\} .
$$

For $v \in C^{\infty}(U)$ and $t>0$, let

$$
\begin{aligned}
& \sigma_{t}(v)=\sup \left\{\|\partial U(Y) v\| t^{n} / n ! \mid Y \in\{X\}^{n}, n \in N\right\} \\
& \tau_{t}(v)=\sup \left\{\left\|\partial U\left(X^{n}\right)\right\| t^{|n|} / n ! \mid n \in N^{d}\right\} .
\end{aligned}
$$

Recall ((I.1) and (I.3) of [9]) that $v \in \mathcal{H}_{\infty}^{\omega}$ iff $\sigma_{t}(v)$ (or equivalently $\tau_{t}(v)$ ) is finite for all $t>0$.

Proposition (I.1). $\mathcal{H}_{\infty}^{\omega}(U)$ is a Fréchet space and $U^{\omega}$ is a bolomorphic representation of $G_{c}$ in $\mathcal{H}_{\infty}^{\omega}$ by continuous operators. Furtbermore, the topologies on $\mathcal{H}_{\infty}^{\omega}$ defined via the families of seminorms $\left\{\sigma_{t}\right\}$ and $\left\{r_{t}\right\}$ both agree with the $\mathcal{H}_{\infty}^{\omega}$ topology.

Proof. If $K_{n}$ is any nested sequence of compact subsets of $G_{c}$ for which $K_{n} \subset K_{n+1}$ and $\bigcup_{n=1}^{\infty} K_{n}=G_{c}$, then $\rho_{K_{n}}$ form a basis of seminorms for $H_{\infty}^{\omega}$. Hence $\mathcal{H}_{\infty}^{\omega}$ is countably normed and is a metric space.

If $v_{n}$ is Cauchy in $\mathcal{H}_{\infty}^{\omega}$ then $z \rightarrow U_{z} v_{n}$ converges (as a map of $G_{c}$ into $\mathcal{H}$ ) uniformly on compacta to a holomorphic function $\phi(z)$. It is easily seen that $v_{n} \rightarrow \phi(e)$ in $\mathcal{H}_{\infty}^{\omega}$. Thus $\mathcal{H}_{\infty}^{\omega}$ is a Fréchet space. 
It is easily verified that $H_{\infty}^{\omega}$ is also a Fréchet space in the topologies defined by the $\left\{\sigma_{t}\right\}$ and $\left\{\tau_{t}\right\}$. The equality of the various topologies then follows from the closed graph theorem and the fact that the injection $\mathcal{H}_{\infty}^{\omega} \rightarrow \mathcal{H}$ is continuous in each topology.

That $U_{z}^{\omega}$ is a continuous operator follows easily from the definitions. To show that $U^{\boldsymbol{z}}$ is holomorphic in $\mathcal{H}_{\infty}^{\omega}$, it suffices, in virtue of (I.1) of [9], to show that $\left\{\partial U(Y) v t^{n} / n ! \mid Y \in\{X\}^{n}, n \in N\right\}$ is bounded in $\mathcal{H}_{\infty}^{\omega}$ for all $t>0$ and all $v \in \mathcal{H}_{\infty}^{\omega}$. This set, however, is clearly bounded in each $\sigma_{s^{\prime}} s>0$, and hence in $\mathcal{H}_{\infty}^{\omega}$, thus proving the analyticity. Q.E.D.

Remarks. The equality of the topologies could also be shown directly from the estimates in $\$ I$ of [9]. Note also that we have so far used only the Banach property of $\mathcal{H}$.

Yet another description of the $\mathcal{H}_{\infty}^{\omega}$ topology is possible.

Definition (1.2). Let $s \in \mathbf{R}^{d}, s=\left(s_{1}, \cdots, s_{d}\right), s_{i}>0$. Let $\mathcal{H}(U)=H^{s}=$ $\left\{v \in \mathcal{H} \mid t \rightarrow U_{\exp t X_{i}} v\right.$ is extendible to a continuous map of $|z| \leq s_{i}$ which is holomorphic on $|z|<s_{i}$ for $\left.i=1, \cdots, d\right\}$. Define

$$
(v, w)_{s}=\sum_{i=1}^{d}\left\{\left(U_{\exp i s_{j}} X_{j} v, U_{\exp i s_{j} X_{j}}^{w)}+\left(U_{\exp -i s_{i} X_{j}} v, U_{\exp -i s_{j} X_{j}}{ }_{j}\right)\right\} .\right.
$$

Let $\|v\|_{s}^{2}=(v, v)_{s}$.

Proposition (I.3). Under \|\|$_{s}, \mathcal{H}^{s}$ is a Hilbert space. Also $\mathcal{H}_{\infty}^{\omega}=$ $\bigcap_{s_{i}>0} \mathcal{H}^{s}$ and the topology of $\mathcal{H}_{\infty}^{\omega}$ is given by the family of restrictions of the norms \|\|$_{s}$.

Proof. From the maximum modulus principle and the unitarity of $U$, it follows that

$$
\sup _{|z| \leq s_{j}}\left\|U_{\exp z X_{j}} v\right\|^{2} \leq\left\|U_{\exp i s_{j} X_{j}} v\right\|^{2}+\left\|U_{\text {exp }-i s_{j} X_{j}} v\right\|^{2}
$$

for $v \in \mathcal{H}^{s}$ and $j=1, \cdots, d$. The completeness of $\mathcal{H}^{s}$ follows from this.

That $\cap \mathcal{H}^{s}=\mathcal{H}_{\infty}^{\omega}$ follows from (I.5) of [9]. The topological part is another application of the closed graph theorem. Q.E.D.

The above proposition yields a characterization of $\left(\mathcal{H}_{\infty}^{\omega}\right)^{\prime}$. If $s=$ $\left(s_{1}, \cdots, s_{d}\right) \in \mathbb{R}^{d}, s_{i}>0$, let $U_{s}: \mathcal{H}^{2} s \rightarrow \mathcal{H}$ be given by

$$
U_{s}=\sum_{j=1}^{d}\left\{U_{\exp _{i} 2 s_{j} X_{j}}+U_{\exp -i 2 s_{j} X_{j}}\right\} \text {. }
$$

Also, if $r, t \in R^{d}$, we will say $t>r$ iff $t_{i}>r_{i}$ for $i=1, \cdots, d$.

Theorem (I.4). Given $\phi \in\left(\mathcal{H}_{\infty}^{(\omega)}\right)^{\prime}$, there is an $s_{0} \in \mathbf{R}^{d}, s_{0}>0$, with the following property: For all $s>s_{0}$ there is a $w_{s} \in \mathcal{H}^{(s}$ which represents $\phi$ in the 
sense that $\phi(v)=\left(U_{s} v, w_{s}\right)$ for all $v \in \mathcal{H}_{\infty}^{\omega}$.

Proof. Note that if $w, v \in \mathcal{H}^{s}$, then, by uniqueness of analytic continuation, $\left(U_{\exp z X_{j}}{ }^{v}, w\right)=\left(v, U_{\text {exp }-\dot{z} X_{j}}{ }^{w)}\right.$ for all $|z| \leq s_{j}$. It follows that, for $v \in \mathcal{H}_{\infty}^{\omega}$ and $w \in \mathcal{H}^{s},\left(U_{s} v, w\right)=(v, w)_{s}$. Thus, our assertion is that there is an $s_{0}$ for which $\phi$ is continuous in \|\|$_{s}^{s}$ for all $s>s_{0}$. This follows from (I.3) and its proof. Q.E.D.

Corollary (I.5). A subspace $M$ of $\mathcal{H}_{\infty}^{\omega}$ is dense iff there is a sequence $s^{p} \epsilon$ $\mathbf{R}^{d}$ for which $s_{i}^{p} \rightarrow \infty$ as $p \rightarrow \infty, i=1, \cdots, d$, and for which $U_{s}{ }^{(M)}$ is dense in $U_{s}{ }_{p}\left(\mathcal{H}_{\infty}^{\omega}\right)$ in the topology of $\mathcal{H}$ for all $p \in N$.

Proof. The usual annihilator argument.

Corollary (I.6). If $\mathcal{H}$ is separable, so is $\mathcal{H}_{\infty}^{\omega}$.

Proof. Let $v_{n}, n=1, \cdots$, be a countable dense subset of $\mathcal{H}$. For $n, m \in N$, $s \in \mathbf{R}^{d}$ if there is a point $w \in \mathcal{H}_{\infty}^{\omega}$ for which $\left\|v_{m}-U_{s} w\right\|<1 / n$, let $w_{s}^{n, m}$ be such a point. Otherwise let $w_{s}^{n, m}=0$. Let $W_{s}=\left\{w_{s}^{n, m} \mid n, m \in N\right\}$ and let $M=$ span $U_{s \in N d^{W}} \cdot U_{s}\left(W_{s}\right)$ is dense in $U_{s}\left(\mathcal{H}_{\infty}^{(\omega)}\right)$ for $s \in N^{d}$. Hence $M$ is dense in $\mathcal{H}_{\infty}^{\omega}$ by (I.S). Finally, the set of linear combinations with complex rational coefficients of elements of $\bigcup_{s \in N d} W_{s}$ is a countable dense subset of $\mathcal{H}_{\infty}^{\omega}$. Q.E.D.

Remarks. In view of the proof of Theorem (II.2) of [9], we might expect that one could produce a countable dense set of entire vectors via regularization - i.e. vectors of the form $\int_{G} \phi(g) U_{g} v d g$ where $\phi(g)$ is an $L^{1}$ entire vector. Although this definitely seems possible, we are unable to prove that the set of such vectors is dense in $\mathcal{H}_{\infty}^{\omega}$. The difficulty is that the integral does not seem to converge in $\mathcal{H}_{\infty}^{\omega}$. It is for this reason, also, that we are unable to prove that $U^{\omega}$ is topologically irreducible if $U$ is (cf. Example (8.20) of [10]).

(I.4) also provides, in the case that $U$ is given as a direct integral of other representations, another description of $\left(\mathcal{H}_{\infty}^{\omega}\right)^{\prime}$.

This description is based on the following fundamental theorem of Goodman's [4, Lemma (3.1)].

Theorem (A.1). If $U=\int_{M} \bigoplus U^{a} d a$ where $M$ is an analytic Borel space and $U^{\alpha}$ is an integrable family of unitary representations of $G$ in $\mathcal{H}^{\alpha}$, then $v \in \mathcal{H}, v=$ $\left\{v^{\alpha}\right\}$ is in $\mathcal{H}_{\infty}^{\omega}(U)$ iff $v^{\alpha} \in \mathcal{H}_{\infty}^{\omega}\left(U^{a}\right)$ for a.e. a and, for all compact sets $\Omega \subset G_{c^{\prime}}$, $\alpha \rightarrow \sup _{z \in \Omega}\left\|U_{z}^{\alpha} v^{\alpha}\right\|^{2}$ is in $L^{1}(M)$.

In this case, $\left(U_{z} v\right)^{a}=U_{z}^{a} v^{a}$ for all $z \in G_{c}$ and a.e. $a$.

If the representation space of $U$ is separable then the same statement, more or less, is true for $\left(H_{\infty}^{\omega}\right)^{\prime}$. 
Proposition (I.7). In the notation of (A.1), if $\phi \in \mathcal{H}_{\infty}^{\omega}(U)^{\prime}$ then for a.e. a there is a uniquely determined functional $\phi^{\alpha} \in \mathcal{H}_{\infty}^{\omega}\left(U^{a}\right)^{\prime}$ for which $\phi(v)=$ $\int_{M} \phi^{\alpha}\left(v^{\alpha}\right) d a$ for all $v=\left\{v^{\alpha}\right\} \in \mathcal{H}_{\infty}^{\omega}(U)$. The integral is absolutely convergent.

Conversely, if $\phi^{a} \in \mathcal{H}_{\infty}^{\omega}\left(U^{a}\right)^{\prime}$ are such that $a \rightarrow \phi^{\alpha}\left(v^{\alpha}\right)$ is in $L^{1}(M)$ for all $v=\left\{v^{\alpha}\right\} \in \mathcal{H}_{\infty}^{\omega}(U)$, then $v \rightarrow \int_{M} \phi^{\alpha}\left(v^{\alpha}\right)$ da defines an element of $\mathcal{H}_{\infty}^{\omega}(U)^{\prime}$.

Proof. The first part, except for uniqueness, follows from (I.4) and (A.1). The uniqueness follows as in (C.1) of [8] except that we now obtain the required countable dense subset of $\mathcal{H}_{\infty}^{\omega}\left(U^{\alpha}\right)$ via the following lemma.

Lemma. If $\left\{v_{n}\right\}_{n=1}^{\infty}$ is dense in $\mathcal{H}_{\infty}^{\omega}(U)$, then for a.e. $\alpha \in M,\left\{v_{n}^{\alpha}\right\}_{n=1}^{\infty}$ is dense in $\mathcal{H}_{\infty}^{\omega}\left(U^{\alpha}\right)$.

Proof. Let $s^{p} \in \mathbf{R}^{d}, p \in N$, be a sequence satisfying the hypothesis of (1.5). Let $K^{p}=U_{s} p\left(\mathcal{H}_{\infty}^{\omega}(U)\right)$ and let $K_{\alpha}^{p}=U_{s}^{a}\left(\mathcal{H}_{\infty}^{\omega}\left(U^{\alpha}\right)\right)$. If $E \subset M$ is measurable, let $\Pi_{E}: \mathcal{H} \rightarrow \mathcal{H}^{s^{p}}$ be the map that takes $\left\{v^{\alpha^{s}}\right\}$ onto $\left\{w^{\alpha}\right\}$ where $w^{\alpha}=v^{\alpha}$ if $\alpha \in E$ and is zero otherwise. It follows from (A.1) that $\Pi_{E}$ leaves $K^{p}$ invariant. Hence $\Pi_{E}$ commutes with the projection $\Pi^{p}$ onto $K^{p}$. It follows from Theorem (P. G) of $[6$, p. 92$]$ that $\Pi^{p}$ is a direct integral of projections $\Pi_{a}^{p} . \Pi_{a}^{p}$ is the projection onto $K_{\alpha}^{p}$ and, hence $K^{p}$ is the direct integral of the $K_{a}^{p}$.

But $U_{s}{ }^{p}\left(\left\{v_{n}\right\}_{n=1}^{\infty}\right)$ is dense in $K^{p}$. Thus $U_{s}^{a}\left(\left\{v_{n}^{\alpha}\right\}_{n=1}\right)$ is, for a.e. $a$, dense in $K_{a}^{p}$. Upon choosing a set of a for which this is true for all $p \in N$, the lemma follows from (I.5).

The converse statement of (I.7) follows from the closed graph theorem as in [8]. Q.E.D.

Corollary (I.8). If in (I.7) each $U^{a}$ is finite dimensional, then $\phi$ is given via a function $\alpha \rightarrow w^{\alpha} \in \mathcal{H}^{\alpha}$ in the sense that $\phi(v)=\int_{M}\left(v^{\alpha}, w^{\alpha}\right) d \alpha$ for all $v \epsilon$ $\mathfrak{H}_{\infty}^{\omega}(U)$.

Furtbermore $\alpha \rightarrow w^{\alpha}$ represents an element of $\mathcal{H}_{\infty}^{\omega}(U)^{\prime}$ iff $\alpha \rightarrow\left(v^{\alpha}, w^{\alpha}\right)$ is integrable for all $\left\{v^{\alpha}\right\} \in \mathcal{H}_{\infty}^{\omega}(U)$.

Example (1.9). Let $G=\mathbf{R}$ and let $U$ be the regular representation. $\mathbf{R}_{c}$ is C and $\mathcal{H}_{\infty}^{\omega}(U)$ is the space of entire functions $f$ on $\mathbf{C}$ for which

$$
\sup _{|y| \leq \delta} \int_{-\infty}^{\infty}|f(x+i y)|^{2} d x<\infty
$$

for all $\delta>0$ (cf. [3, p. 64]).

By the Paley-Wiener theorem this is the space of functions which, when restricted to the real line, satisfy $\int_{-\infty}^{\infty}|\hat{f}(\lambda)|^{2} \exp \{2 \delta|\lambda|\} d \lambda<\infty$ for all 
$\delta>0$. ( $~$ indicates Fourier transform.)

defines a direct integral decomposition of $U$ into one dimensional representations. (I.8) applies and shows that, to each element $\phi$ of $\left(\mathcal{H}_{\infty}^{\omega}\right)^{\prime}$, there is a unique function $\phi: \mathbf{R} \rightarrow \mathbf{C}$ for which $\phi(f)=\int_{-\infty}^{\infty} \hat{f}(\lambda) \hat{\phi}(\lambda) d \lambda$. The space of such $\hat{\phi}$ can be characterized as the set of functions for which $|\hat{\phi}(\lambda)| \leq e^{\delta|\lambda|}$ for some $\delta>0$ (depending on $\phi$ ). $\mathcal{H}_{\infty}^{\omega}$

One of our main uses of (I.3) will be in the study of the duality theory of

Definition (I.10). For $w \in \mathcal{H}_{\infty}^{\omega}$ and $z \in G_{c}$ define the seminorm \|\|$_{z, w}$ by $\|v\|_{z, w}=\left|\left(U_{z} v, u\right)\right|$ for $v \in \mathcal{H}_{\infty}^{\omega}$. Let $\mathbb{C}_{\infty}^{\omega}=\mathfrak{l}_{\infty}^{\omega}{ }_{\infty}^{c}(U)$ be $\mathcal{H}_{\infty}^{\omega}$ with the topology defined by the family $\|\cdot\|_{z, w}$.

Proposition (I.11). $\mathbb{C}_{\infty}^{\omega}$ is $\mathcal{H}_{\infty}^{\omega}$ with its weak topology.

Proof. (I.4).

We shall also need an intermediary topology.

Definition (I.12). If $\Omega \subset G_{c}, \Omega$ compact, let $\|v\|_{\Omega, w}=\sup _{z \in \Omega}\|v\|_{z, w}$ for $v, w \in \mathcal{H}_{\infty}^{\omega}$. Let $\Re_{\infty}^{\omega}$ be $\mathcal{H}_{\infty}^{\omega}$ with the topology defined via the family $\|\cdot\|_{\boldsymbol{\Omega}, \boldsymbol{w}}^{\boldsymbol{z}}$.

Recall that a locally convex topological vector space $\mathcal{F}$ is said to be semiMontel if all closed and bounded sets are compact. If $\mathcal{F}$ is also barrelled, $\mathcal{F}$ is said to be Montel.

Proposition (I.13). $\mathfrak{M}_{\infty}^{\omega}$ and $\mathrm{C}_{\infty}^{\omega}$ are semi-Montel spaces.

Proof. By the uniform boundedness principle in $\mathcal{H}_{\infty}^{\omega}, \mathfrak{H}_{\infty}^{\omega}, \mathcal{H}_{\infty}^{\omega}$, and $\mathbb{T}_{\infty}^{\omega}$ all have the same bounded sets. Since the injection $\mathbb{\pi}_{\infty}^{\omega} \rightarrow \mathbb{L}_{\infty}^{\omega}$ is continuous, it suffices to prove the assertion for $\mathbb{M}_{\infty}^{\omega}$.

Let $\left\{v_{\alpha}\right\}_{a \in A}$ be a bounded net in $\pi_{\infty}^{\omega}$. Then $\left\{v_{a}\right\}_{a \in A}$ is bounded in $\mathcal{H}$. Hence we may assume $v_{\alpha}$ converges weakly to some element $v \in \mathcal{H}$. Now, let $w \in \mathcal{H}$ and set $\phi_{w}(z)=\sup _{\alpha \in A}\left|\left(U_{z} v^{\alpha}, w\right)\right|, z \in G_{c}$. Let $\Omega_{w}$ be the space of entire functions on $G_{c}$ which are dominated by $\phi_{w}$. From the boundedness of $v^{a}$, $\phi_{w}$ is bounded on compact subsets. Hence $\Omega_{w}$ is a closed bounded subset of the space of entire functions with the compact open topology. By Montel's theorem $\Omega_{w}$ is compact. Let $\Omega=\Pi_{w \in H} \Omega_{w}$. By the Tychonoff theorem $\Omega$ is compact. Let $\left\{X_{\alpha}\right\}_{\alpha \in A} \in \Omega$ be the net $X_{\alpha}^{w}=z \rightarrow\left(U_{z} \nu^{\alpha}, w\right)$ and let $\left\{X_{n_{\beta}}\right\}_{\beta \in A^{\prime}}$ be a convergent subnet, say $X_{n \beta} \rightarrow X$. Then $X_{n_{\beta}}^{w} \rightarrow X^{w}$ uniformly on compact subsets of $G_{c}$ for all $w \in \mathcal{H}, X^{w}$ is an entire function and, for $g \in G, X^{w}(g)=$ $\lim _{\beta \in A},\left(U_{8}^{c} v_{n}, w\right)=\left(U_{8} v, w\right)$. Thus, by (1.2) of [9], $v \in \mathcal{H}_{\infty}^{\omega}$ and $v_{n_{\beta}} \rightarrow v$ in $\pi_{\infty}^{\omega} \omega^{\circ}$ Q.E.D.

Corollary (I.14). $\mathcal{H}_{\infty}^{\omega}$ is reflexive. 
Proof. Since $\mathbb{W}_{\infty}^{\omega}$ is semi-Montel, $\mathcal{H}_{\infty}^{\omega}$ is weakly reflexive by Proposition 1 of $\left[5\right.$, p. 227]. $\mathcal{H}_{\infty}^{\omega}$ is barrelled since it is Fréchet. Our conclusion then follows from Proposition 6 of [5, p. 229]. Q.E.D.

Remark. The above proposition is actually true for any representation of $G$ in a reflexive Fréchet space. The same proof carries over with only minor changes.

Corollary (I.15). The relative topologies gotten by restricting $\pi_{\infty}^{\omega}$ and $70_{\infty}^{\omega}$ to a fixed bounded set agree. In particular a sequence $x_{n}$ converges in $\pi_{\infty}^{\omega}$ iff it converges in $\mathfrak{C}_{\infty}^{\omega}$.

Proof. It suffices to consider only the $\pi_{\infty}^{\omega}$ bounded sets, in which case the proposition follows from compactness. Q.E.D.

In general, the topology of $\mathcal{H}_{\infty}^{\omega}$ cannot be any nicer than that of $\mathcal{H}$ since if $U$ is uniformly continuous, for example, $\mathcal{H}_{\infty}^{\omega}=\mathcal{H}$. However; if $G$ is nilpotent and $U$ is irreducible, the topology is, in some sense, significantly nicer.

Theorem (I.16). If $G$ is nilpotent and $U$ is irreducible, then $\mathcal{H}_{\infty}^{\omega}$ is a Montel space.

Before proving (I.16), we comment that we do not know the most general type of group $G$ for which the theorem is true. However (I.16) implies that $G$ is C.C.R. for, if $f$ is any entire vector for the $L^{1}$ left regular representation of $G$, then $U_{f}=\int_{G} f(g) U_{g} d g$ maps $\mathcal{H}$ into $\mathcal{H}_{\infty}^{\omega}$. By the closed graph theorem $U_{f}$ is continuous. From the continuity of the injection $\mathcal{H}_{\infty}^{\omega} \rightarrow \mathcal{H}$, it follows that $U_{f}$, as a map of $\mathcal{H}$ into $\mathcal{H}$, is a compact operator if $\mathcal{H}_{\infty}^{\omega}$ is a Montel space. Finally, every operator $U_{b}, b \in L^{1}(G)$, is a uniform limit of such $U_{f}$. Hence every such $U_{b}$ is compact and $G$ is C.C.R.

Auslander-Moore [1, Chapter V] have shown that, for type $R$ groups, C.C.R. is equivalent to type I. We suspect that $(1.16)$ is also.

Proof of (1.16). Let $B$ be closed and bounded in $\mathcal{H}_{\infty}^{\omega}$. To show that $B$ is compact it suffices, we claim, to show that $U_{z} B$ is compact in $\mathcal{H}$ for all $z \in G_{c^{\prime}}$ for in this case $B=\Pi_{z \in G_{c}} U_{z} B$ is compact. Hence every net $\left\{x_{\alpha}\right\}_{a \in A}$ in $B$ has a subnet $\left\{y_{\beta}\right\}_{\beta \in A}$, for which $U_{z} y_{\beta}$ converges in $\mathcal{H}$ for all $z \in G_{c}$. By (I.3) $y_{\beta}$ then converges in $\mathcal{H}_{\infty}^{\omega}$. Thus, it suffices to show that every $\mathcal{H}_{\infty}^{\omega}$ bounded set $B$ is compact in $\mathcal{H}$.

Now in [12], Pukánszky showed (Part II, Chapter II, Theorem 2 and its proof) that since $U$ is irreducible, there is an element $X \in \mathcal{U}(G)$ for which $\partial U(X)$ has a bounded inverse $T$. From the eigenvalues for $T$ as computed in [12], $T$ is a compact operator. Hence $B=T \partial U(X) B$ is a compact subset of H. Q.E.D. 
II. Function spaces. We are, in this section, interested in representations realized in spaces of functions on $G$ as defined below.

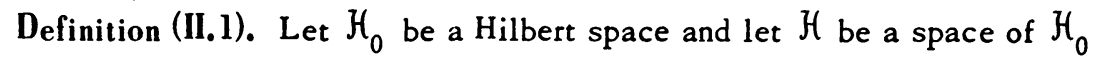
valued locally integrable functions (with a.e. equal functions identified) with respect to Haar measure. Suppose $\mathcal{H}$ is topologized in such a manner that:

(1) $\mathcal{H}$ is a Banach space.

(2) The injection of $\mathcal{H}$ into $L_{\text {loc }}^{1}\left(G, \mathcal{H}_{0}\right)$ is continuous.

(3) For all $f \in \mathcal{H}$ and $g \in G$, the function $R_{g} f: x \rightarrow f(x g)$ is in $\mathcal{H}$ and $g \rightarrow$ $R_{g}$ defines a continuous representation of $G$ in $\mathcal{H}$.

Then $\mathcal{H}$ is said to be a regular Banacb space of $\mathcal{H}_{0}$-valued functions and $R$ is said to be the regular representation of $G$ in $\mathcal{H}$.

If $\mathcal{S}$ is any Fréchet space and $F: G \rightarrow \mathcal{J}$ is a $C^{\infty}$ function then define, for $X \in \mathfrak{d}$,

$$
\tilde{X} F(g)=\lim _{t \rightarrow 0} \frac{F(g \exp t X)-F(g)}{t} .
$$

In this terminology, we have the following generalization of results of Goodman [4] and Poulsen [11].

Proposition (II.2). Let $f \in \mathcal{H} . f \in C^{\infty}(R)$ iff $f$ is a $C^{\infty} \mathcal{H}_{0}$ valued function and $\tilde{X}_{1} \ldots \tilde{X}_{n} f \in \mathcal{H}$ for all $X_{1}, \cdots, X_{n} \in \mathcal{L}$.

$f \in \mathcal{H}_{\infty}^{\omega}(R)$ iff $f$ is extendible to an entire $\mathcal{H}_{0}$ valued map of $G_{c}$ for which the maps $R_{, z} f: g \rightarrow f(g z)$ are in $\mathcal{H}$ for all $z \in G_{c}$ and $z \rightarrow R_{z} f$ is continuous in $\mathcal{H}$.

Proof. Suppose $f$ satisfies the hypothesis of the "only if" part.of the above. For each $t \in \mathbf{R}$ and $X \in \mathscr{L}(G)$ let $g_{t}=\int_{0}^{t} R(\exp t X) X f d t$. (This $\mathcal{H}$ valued integral exists by Lemma 2, p. 12 of [7].) Let $w \in\left(\mathcal{H}_{0}\right)^{\prime}$ and let $\phi \epsilon$ $\mathscr{D}(G)$. By evaluating with functionals of the form

$$
g \rightarrow \int_{G} \phi(x)\langle g(x), w\rangle d x \quad(g \in \mathcal{H})
$$

it is easily seen that $g_{t}=R(\exp t X) f-f$. It follows that $f$ is a $C^{1}$-vector and hence, by induction, that $f$ is a $C^{\infty}$-vector. Conversely, if $f$ is a $C^{\infty}$-vector for $R$ and $w \in\left(\mathcal{H}_{0}\right)^{\prime}$, then it follows by evaluating $\partial R(X) f$ with functionals of the above form that $x \rightarrow\langle\partial R(X) f(x), w\rangle$ is the distributional derivative (along $X)$ of $x \rightarrow\langle f(x), w\rangle$. It follows by induction and the Sobolev theorem that $x \rightarrow\langle f(x), w\rangle$ is a $C^{\infty}$-function. Since weak $C^{\infty}$ implies strong $C^{\infty}$, we get that $f$ is a $C^{\infty} \mathcal{H}_{0}$ valued map (see [7, Lemma 1 , p. 47]).

To prove the entirety part, let $f \in \mathcal{H}_{\infty}^{\omega}(R)$. Then $f$ is, in particular, a $C^{\infty}$. vector and hence is continuous. Let $K$ be a compact neighborhood of $e$ in $G$ and let $\mathcal{C}\left(K, \mathcal{H}_{0}\right)$ be the Banach space of continuous $\mathcal{H}_{0}$ valued functions on 
$K$ given the sup-norm topology.

The restriction map $\left.f \rightarrow f\right|_{K}$ of $\mathcal{H}_{\infty}^{\omega}(R)$ into $\mathcal{C}\left(K, \mathcal{H}_{0}\right)$ is continuous by the closed graph theorem and the regularity of $\mathcal{H}$. It follows that point evaluation is a continuous linear transform on $\mathcal{H}_{\infty}^{\omega}(R)$. We conclude from (I.1) above that $z \rightarrow$ $\left(U_{z}^{\omega} f\right)(e)$ is a holomorphic function on $G_{c}$. This is the desired extension. Clearly $U^{\omega}$ acts as claimed.

Finally, if $f$ has an extension satisfying the above hypothesis, let $\gamma$ be a closed curve in $G_{c}$. By evaluating with the appropriate functionals (as in the $C^{\infty}$ part), it is easily shown that $\int_{y} R_{z} f d z \in \mathcal{H}$ is zero. Hence Morera's theorem finishes the proof.

Corollary (II.3). Let $R$ be the unitary representation of $G$ induced from $a$ unitary representation $L$ of a closed subgroup of $G$. If $U$ is realized as in Blattner [2], then $R$ is a regular representation and the characterization of (II.2) applies.

Corollary (II.4). Let $\mathcal{H}_{0}=\mathrm{C}, \mathcal{H}=L^{p}(G)(1 \leq p \leq \infty)$. Let $R$ be the right regular representation of $G$ in $\mathcal{H}$. Then (II.2) applies and we obtain the expected characterization of $\mathcal{H}_{\infty}^{\omega}(R)$.

Remark (II.2) is a generalization of results from [11]. (II.4) in the nilpotent case is due to Goodman [4].

Remark (II.5). Note that in the above proof we showed that $f \rightarrow f(e)$ is continuous from $\mathcal{H}_{\infty}^{\omega}(R)$ into $\mathcal{H}_{0}$. It follows that $f \rightarrow f(z)$ is continuous for all $z \epsilon$ $G$. If $\mathcal{H}_{0}=\mathbf{C}$ we obtain from (I.4) a Cauchy-like representation theorem for $\mathcal{H}_{\infty}^{\omega}(R)$. In fact, if $R$ is the right regular representation of $G$, our representation theorem takes the following form:

Corollary. Let $R$ be the $L^{2}$ right regular representation. Then, for all $z \in G_{c}$, there is an $s_{0} \in R^{d}, s_{0}>0$, with the following property: For all $s>s_{0}$ there is a $w_{s}^{z} \in \mathcal{H}^{s}(R)$ such that

$$
f(z)=\sum_{j}\left\{\int_{G} f\left(g \exp \text { is } X_{j}\right) \bar{w}_{s}^{z}(g) d g+\int_{G} f\left(g \exp -i s_{j} X_{j}\right) \bar{w}_{s}^{z}(g) d g\right\}
$$

for all $f \in \mathcal{H}_{\infty}^{\omega}(R)$,

Note, incidentally, that the modular function does not appear in the above formula. The reason is that for type $R$ groups, the trace of the adjoint representation is unity and hence $G$ is unimodular. Type $R$ groups are the only ones whose regular representation has nonzero entire vectors.

In general, the kernels $w_{s}^{z}$ seem to be difficult to compute. For $R$ the right regular representation and $z$ real, it suffices to compute $w_{s}^{e}$, for let $L$ be the $L^{2}$ left regular representation of $G$. Since $L$ and $R$ commute, $L$ leaves $H^{s}(R)$ invariant and $L$ is unitary in \|\|$_{\odot}$. Thus, for $f \in \mathcal{H}_{\infty}^{\omega}(R)$ and $g \in G$, 


$$
f(g)=L_{g^{-1}} f(e)=\left(L_{g^{-1}} f, w_{s}^{e}\right)_{s}=\left(f, L_{g} w_{s}^{e}\right)_{s}
$$

Thus $w_{s}^{g}=L_{g} w_{s}^{e}$.

If $\mathrm{G}=\mathbf{R}$, we may explicitly compute $w_{s / 2}^{e}=w$ as follows: From the PaleyWiener theorem and (II.2), it follows that the Fourier transform $\hat{f}$ of a function $f \in L^{2}(G)$ is in $\mathcal{H}_{\infty}^{\omega}(R)$ iff $\int_{-\infty}^{\infty} e^{a|t|}|f(t)|^{2} d t<\infty$ for all $\alpha \geq 0$. (Cf. Goodman $[3$, p. 64], for details.) The analytic extension of $\hat{f}$ is given by

$$
\hat{f}(z)=(2 \pi)^{-1 / 2} \int_{-\infty}^{\infty} e^{-i z x} f(x) d x .
$$

Hence, letting - denote the conjugate of the inverse Fourier transform

$$
\begin{aligned}
(2 \pi)^{-1 / 2} \int_{-\infty}^{\infty} f(x) d x & =\hat{f}(0)=\int_{-\infty}^{\infty} \hat{f}(x+i s) \bar{w}(x) d x+\int_{-\infty}^{\infty} \hat{f}(x-i s) \bar{w}(x) d x \\
& =\int_{-\infty}^{\infty} 2(\cosh s x) f(x) \check{w}(x) d x .
\end{aligned}
$$

Thus $\check{w}(x)=2(2 \pi)^{-1 / 2} /$ cosh $s x$. The Fourier transform can be explicitly computed via a contour integral to be $w(x)=[4 s \cosh (\pi x / 2 s)]^{-1}$.

III. Applications to complex solvmanifolds. Let $S$ be a solvable, connected, simply connected Lie group (not necessarily type $R$ ) and let $\Gamma$ be a closed but not necessarily connected subgroup. Then the homogeneous space $M=S / \Gamma$ is a solvmanifold. If $S$ is a complex Lie group and $\Gamma$ is a complex Lie subgroup (i.e. the component of the identity of $\Gamma$ is a complex analytic subgroup of $S$ ), then $S / \Gamma$ is a complex manifold and will be called a complex solvmanifold.

Lemma (III.1). Let $\Gamma_{0}$ be the component of the identity of $\Gamma$ and let $\left(\Gamma_{0}\right)_{c} \subset S_{c}$ be its complexification. Let $\Gamma_{c}=\Gamma \cdot\left(\Gamma_{0}\right)_{c}$. Then $\Gamma_{c}$ is a closed, complex Lie subgroup of $S_{c}$ and $M$ is canonically imbedded in $M_{c}=S_{c} / \Gamma_{c}$.

Proof. There is a Jordan-Hölder basis $B$ of $\mathfrak{L}(S)$ which contains a JordanHölder basis $B_{0}$ of $\mathscr{L}\left(\Gamma_{0}\right)$. Letting $B$ define holomorphic coordinates for $S_{c}$ as in the proof of (I.1) of [9], one sees that $B_{0}$ defines holomorphic coordinates for $\left(\Gamma_{0}\right)_{c}$ and hence $\left(\Gamma_{0}\right)_{c} \cap S=\Gamma_{0}$. Let $\Gamma_{c}=\Gamma \cdot\left(\Gamma_{0}\right)_{c}$.

We claim that $\left(\Gamma_{0}\right)_{c}$ is invariant under $\Gamma$ and hence that $\Gamma_{c}$ is a subgroup. To see this let \|\| be a complex norm on $\mathscr{L}_{c}(S)$ and let $\mathcal{U}$ be a closed ball in $\mathscr{L}_{c}(S)$ such that

(i) exp is a homeomorphism onto the image $U$ of $\mathcal{U}$ in $S_{c}$, and

(ii) $\mathcal{U}$ is sufficiently small in the sense defined below.

For $z \in S_{c}$ and $\alpha \in C,|\alpha| \leq 1$, define $\alpha z=\exp (\alpha(\log z))$ wherever $\log z$ is defined and single valued. If $z \in U$ and $U$ is sufficiently small, the elements $y=(-i / 2)\left(\bar{z}^{-1} z\right)$ and $x=z(i y)^{-1}$ are defined and satisfy $z=x(i y)$ (- denotes the canonical conjugation on $S_{c}$ ). Furthermore, it is easily seen that $y=\bar{y}$, $x=\bar{x}$ and, hence, that $y$ and $x$ are in $\left(\Gamma_{0}\right)_{c} \cap S=\Gamma_{0}$. It follows that if $g$ is 
a fixed element of $\Gamma$ and $U$ is small enough (relative to $g$ ), then

$$
g z g^{-1}=\left(g x g^{-1}\right)\left(g(i y) g^{-1}\right)=\left(g x g^{-1}\right)\left(i\left(g y g^{-1}\right)\right) \in\left(\Gamma_{0}\right)_{c} .
$$

Since $U \cap\left(\Gamma_{0}\right)_{c}$ generates $\left(\Gamma_{0}\right)_{c}, g$ leaves $\left(\Gamma_{0}\right)_{c}$ invariant. Hence $\Gamma \cdot\left(\Gamma_{0}\right)_{c}$ is a subgroup.

It follows similarly that $\Gamma_{c}$ is closed since to show closure it suffices to show that there is a closed neighborhood $U$ of $e$ in $S_{c}$ such that $U \cap \Gamma$ is closed. If $U$ is sufficiently small we can work with real and imaginary parts as above.

The map $S / \Gamma \rightarrow S \cdot \Gamma_{c} / \Gamma_{c} \subset M_{c}$ is easily seen to be an imbedding (since $\Gamma_{c} \cap S=\Gamma$ ) and the lemma follows. Q.E.D.

We are interested in the following questions about $M_{c}$.

(i) Under what conditions does the set of entire functions $\Omega\left(M_{c}\right)$ separate points of $M$ ?

(ii) When does $\Omega\left(M_{c}\right)$ separate points of $M_{c}$ ?

Our answers are as follows:

Theorem (III.2). Supopse $\Gamma$ contains no nontrivial normal analytic subgroups. Then:

(i) If $S$ is type $R$, then $\Omega\left(M_{c}\right)$ separates points of $M$. If $S / \Gamma$ is compact, it is also necessary that $S$ be type $R$ for point separating to bold.

(ii) If $S$ is type $R$, then there is a closed complex subgroup $\Gamma^{\prime} \subset S_{c}$ with the following properties:

(a) $\Gamma^{\prime} \supset \Gamma_{c}$ and $\left(\Gamma^{\prime}\right)_{0}=\left(\Gamma_{c}\right)_{0}=\left(\Gamma_{0}\right)_{c}$.

(b) $\Gamma^{\prime} \cap S=\Gamma$ and there is a natural imbedding of $M$ into $S_{c} / \Gamma^{\prime}$.

(c) $\Omega\left(S_{c} / \Gamma^{\prime}\right)$ separates points of $S_{c} / \Gamma^{\prime}$.

l.e., if we change our notion of complexification slightly, $M$ bas a complexification for which the bolomorphic functions separate points.

Proof. (i) Let $S$ be type $R$. Let $C_{0}(M)$ be the Banach space all continuous functions vanishing at infinity given the sup norm. We may identify $C_{0}(M)$ with a space of functions $\mathcal{C}$ on $G$ which are invariant under right translation by elements of $\Gamma$. $S$ acts on $\mathcal{C}$ via left translation and this action defines a representation $R$ of $S$ in $C$. $C$ is a regular Banach space of complex functions (in the sense of II.1) and $R$ is the left regular representation of $S$ in $C$. By (II.3) of [9], $R$ has a dense set of entire vectors and by (II.2) above each entire vector $f$ extends to an entire function $f_{c}$ on $S_{c}$. Since $f$ is invariant under $\Gamma, f_{c}$ is invariant under $\left(\Gamma_{0}\right)_{c}$ (by uniqueness of analytic extensions in $\left(\Gamma_{0}\right)_{c}$ ) and hence under $\Gamma_{c}$. Thus, upon projection $f_{c}$ defines an element $\tilde{f}_{c}$ of $\Omega\left(M_{c}\right)$. The set of such $\tilde{f}_{c}$ separate points of $M$ since they are dense in $C_{0}(M)$. 
If $S / \Gamma$ is compact, but not necessarily type $R$, let $L$ denote the left regular representation of $S$ in $L^{2}(S / \Gamma)$.

It follows from (II.2) and the above reasoning that $\mathcal{H}_{\infty}^{\omega}(L)$ can be identified with a subspace of $\Omega\left(M_{c}\right)$. In fact, since $M$ is compact, it is easily seen that $\boldsymbol{\Omega}\left(M_{c}\right)=\mathcal{H}_{\infty}^{\omega}(L)$. It follows from (II.4) of [9] that every element of $\Omega\left(M_{c}\right)$ is left fixed by left translation by elements of the Green kernel $K$ of $S$ (recall that the Green kemel is the smallest analytic normal subgroup $K$ for which $S / K$ is type $R$ ). In particular, every element of $\Omega\left(M_{c}\right)$ is constant on $K$. If $\Omega\left(M_{c}\right)$ separates points of $M$, then $K \subset \Gamma$. Hence $K=\{e\}$ and $S$ is type $R$.

(ii) Let $\pi: S_{c} \rightarrow M_{c}$ be the projection map and let $\Gamma^{\prime}=\left\{z \in S_{c} \mid f(\pi(z))=\right.$ $\left.f(\pi(e)) \forall f \in \Omega\left(M_{c}\right)\right\}$. $\Gamma^{\prime}$ is a closed subgroup of $S_{c}$ which contains $\Gamma$. We claim that $\Gamma^{\prime}$ is a complex subgroup. To see this, let $Z \in \mathscr{L}\left(S_{c}\right)$ be such that $\exp t Z \in \Gamma^{\prime}$ for all $t \in R$.

If $f \in \Omega\left(M_{c}\right)$, then the map $w \rightarrow f(\pi(\exp w Z))$ of $\mathbf{C}$ into $\mathbf{C}$ is holomorphic and is constant on $\mathbf{R}$. Hence it is constant and, in particular $f(\pi(\exp i t Z))=f(\pi(e))$ for all $t \in R$. Thus $i Z \in \mathscr{L}\left(\Gamma^{\prime}\right)$, as claimed.

Similarly, it follows that $\Gamma^{\prime}$ is invariant under the canonical conjugation in $S_{c}$. Therefore $\mathscr{L}\left(\Gamma^{\prime}\right)$ is the complexification of a real Lie subalgebra of $S$. Since $\Gamma^{\prime} \cap S=\Gamma$ (since $\Omega\left(M_{c}\right)$ separates points of $S / \Gamma$ ), it follows that $\mathfrak{L}\left(\Gamma^{\prime}\right)$ is the complexification of $\mathscr{L}(\Gamma)$ and hence that $\left(\Gamma^{\prime}\right)_{0}=\left(\Gamma_{0}\right)_{c}$, as claimed. The rest of the proposition follows. Q.E.D.

Remarks. We know of no examples where $\Gamma^{\prime} \neq \Gamma_{c}$. It would be interesting to know if such examples exist.

Acknowledgements. The author would like to thank his thesis advisor, Roe Goodman, for inspiring this work, especially $\$ \$ I$ and II.

\section{BIBLIOGRAPHY}

1. L. Auslander and C. C. Moore, Unitary representations of solvable Lie groups, Mem. Amer. Math. Soc. No. 62 (1966). MR 34 \# 7723.

2. R. Blattner, On induced representations, Amer. J. Math. 83 (1961), 79-98. MR 23 \# A2757.

3. R. Goodman, Analytic and entire vectors for representations of Lie groups, Trans. Amer. Math. Soc. 143 (1969), 55 - 76. MR 40 \# 1537.

4. - Complex Fourier analysis on nilpotent Lie groups, Trans. Amer. Math. Soc. 160 (1970), 373-391.

5. J. Horvath, Topological vector spaces and distributions. Vol. I, AddisonWesley, Reading, Mass., 1966. MR 34 \#4863.

6. G. W. Mackey, The theory of group representations, Lecture Notes (Summer, 1955), Dept. of Math., Univ. of Chicago, Chicago, Ill. MR 19, 117.

7. R. Moore, Measurable, continuous and smooth vectors for semigroups and group representations, Mem. Amer. Math. Soc. No. 78 (1968). MR 37 \# 4669. 
8. R. Penney, Canonical harmonic analysis of Lie groups and a Frobenius reciprocity theorem (to appear).

9. - Entire vectors and holomorphic extensions of representations, Trans. Amer. Math. Soc. (to appear).

10.

Entire vectors for representations of Lie groups, Thesis, M.I.T., Cambridge, Mass., 1971.

11. N. S. Poulsen, $O n C^{\infty}$ vectors and intertwining bilinear forms for representations of Lie groups, J. Functional Analysis 9 (1972), 87-120.

12. L. Pukánszky, Leçons sur les représentations des groupes, Monographies de la Société Mathématique de France, no. 2, Dunod, Paris, 1967. MR 36 \#311.

DEP ARTMENT OF MATHEMATICS, PURDUE UNIVERSITY, LAFAYETTE, INDIANA 47907 